Workshop report

\title{
Finding Opportunities in the Area of Alternative Methods to Animal Testing for Romania and Inauguration of the Romanian Center for Alternative Test Methods (ROCAM)
}

Lucian Farcal ${ }^{1,9}$, Francois Busquet ${ }^{2}$, Sandra Coecke ${ }^{3}$, Ioana Hristescu ${ }^{4}$, Christophe Chesné ${ }^{5}$, Christian Pellevoisin ${ }^{6}$, Adriana Orasanu ${ }^{7}, Z_{\text {Zorita Diaconeasa }}^{8}$, Adrian Oros ${ }^{8}$, Adela Pintea ${ }^{8}$ and Carmen Socaciu ${ }^{8}$

${ }^{1}$ BIOTOX Srl, Cluj-Napoca, Romania; ${ }^{2}$ Center for Alternatives to Animal Testing, CAAT-Europe, University of Konstanz, Germany; ${ }^{3}$ DG JRC-Institute for Health and Consumer Protection, European Union Reference Laboratory for Alternatives to Animal Testing (EURL ECVAM), Ispra, Italy; ${ }^{4}$ National Sanitary Veterinary and Food Safety Authority, Bucharest, Romania; ${ }^{5}$ BIOPREDIC International, Rennes, France; ${ }^{6}$ Episkin Academy, Lyon, France; ${ }^{7}$ Institute for Diagnosis and Animal Health, Bucharest, Romania; ${ }^{8}$ University of Agricultural Sciences and Veterinary Medicine, Cluj-Napoca, Romania; ${ }^{9}$ Douglas Connect $\mathrm{GmbH}$, Zeiningen, Switzerland

http://dx.doi.org/10.14573/altex.1509281

On June 5,2015 a workshop on alternative methods to animal testing was held at the University of Agricultural Sciences and Veterinary Medicine (USAMV) in Cluj-Napoca, Romania. The event was organized with the support of the Center for Alternatives to Animal Testing (CAAT-Europe), The European Union Reference Laboratory for alternatives to animal testing (EURL ECVAM) and the National Sanitary Veterinary and Food Safety Authority (ANSVSA), and was sponsored by BIOPREDIC International and Episkin Academy. The main objective was to bring together national and European stakeholders that can play a crucial role in the future developments in this area in Romania, thus the workshop represented a good opportunity to initiate discussions, map existing activities and plan further actions. Around 45 participants with different backgrounds attended the event, e.g., from academia, regulatory bodies and policy-makers, small and medium enterprises (SMEs) as well as non-governmental organizations (NGOs). The event aimed to be a starting point for future developments in the area of 3Rs (Replacement, Reduction and Refinement) in Romania, e.g., to promote and identify educational, research and business opportunities in this field, to disseminate the 3 Rs principles among scientists, to raise awareness on alternative methods applications (i.e., state-of-the-art, in silico, in vitro methods, validation principles and regulatory status), to discuss Directive 2010/63/EU on the protection of animals used for scientific purposes and to learn about the current status and implementation into the Romanian legislation. During the event the Romanian Center for Alternative Test Methods (ROCAM), which is hosted by the USAMV Cluj-Napoca at the Institute of Life Sciences, was established.

The event was divided into four sessions: plenary lectures open to all participants, followed by practical training on an in vitro skin model, a round table discussion and a tour of the research facilities. The plenary lecture session was opened by Prof. Carmen Socaciu (Vice Rector for Research and Innovation at USAMV Cluj-Napoca) with a presentation on past and ongoing activities that involve the use of alternative methods (in vitro), as well as relevant research projects and publications of the group which support the initiative of the university to organize the event and to host the 3Rs Center ROCAM. Dr Francois Busquet (Policy Coordinator at CAAT-Europe and ecopa's Secretary) continued with an extensive presentation on the state-of-the-art of alternative methods in Europe and international trends in the area of the 3 Rs. This was an excellent introduction into the topic for all participants. The presentation included the historical and present circumstances, EU and international frameworks, the list of "3Rs Centers" in Europe as well as information on the impact of alternative method implementation on consumer products related industry. Dr Sandra Coecke (DG JRC-Institute for Health and Consumer Protection, The European Union Reference Laboratory for alternatives to animal testing) presented EURL ECVAM's practices for validation and regulatory acceptance of in vitro methods with the involvement of the European Union Network of 
Laboratories for the Validation of Alternative Methods (EUNETVAL). The legislative background of EURL ECVAM and the EU-NETVAL establishment was shown followed by a presentation of the formal validation process conducted for in vitro methods. Finally, current activities and future challenges for EU-NETVAL were presented. In this context the importance of the involvement of all EU countries within EU-NETVAL, including also Romania which has - so far - no laboratory representative under this framework, was underlined. The plenary session continued with the presentation of Dr Ioana Hristescu (National Sanitary Veterinary and Food Safety Authority) who is the Romanian National Contact Point (NCP) for Directive 2010/63/EU. She described the status of implementation into the Romanian legislation. She also detailed national working practices and governmental agency implementation steps to translate the directive into national law 43/2014. The key points of the new legislation were addressed in this context.

In the second part of the session, examples of advanced in vitro test systems (development and use) were presented. Dr Christian Pellevoisin (Episkin Academy) presented the regulatory context on using in vitro methods for testing of chemicals and consumer products exemplified with 3D skin models validated for this purpose. Furthermore, Dr Christophe Chesné (BIOPREDIC International) presented elements of hepatotoxicity testing based on in vitro models from a scientific and business perspective. Different liver models and approaches were shown in the context of their applications for safety testing or research purposes.

The workshop continued with a training session held by Dr Pellevoisin (Episkin Academy) addressed to PhD students and postdocs. The training included a lecture and a practical demonstration on the use of in vitro SkinEthic Reconstructed Human Epidermis in the skin irritation protocol (OECD TG 439). In the context of ROCAM's role in 3Rs education and training, the organization of similar training sessions in the future was discussed.

The round table session chaired by Dr Lucian Farcal (BIOTOX Srl/Douglas Connect $\mathrm{GmbH}$ ) had the main objective to discuss the role, work program and structure of ROCAM, and also funding opportunities. The proposal was made to structure the activities into Working Groups (WG), e.g., WG on Regulatory Affairs, WG on Education \& Training and WG on Research, in order to effectively coordinate and implement ROCAM's mission. The activities should be coordinated by the Management Team formed by ROCAM's Director together with the WG Coordinators. Moreover, ROCAM will establish an external Scientific Advisory Board in order to follow and advise on its mission and activities. The management tasks will include, e.g., to propose and approve the general strategy and the work program, facilitate the activities of different working groups, to manage the funding, affiliations and agreements, to establish partnerships with national and international stakeholders, to initiate and organize meetings, to prepare the annual report and coordinate the dissemination the activities. A main task of the management team is to identify and further establish a network of 3Rs laboratories in Romania (academia, industry, etc.) that can further support ROCAM in relation with national and international partners. The WG on Regulatory Affairs will offer support on the existing EU legislation (e.g., Directive 2010/63/EU and its correspondent national law No. 43/2014, EU Regulation No. 1223/2009 (Cosmetics), EU Regulation No. 1907/2006 (REACH), EU Regulation No. 528/2012 (Biocidal Products), food and drug related regulations or guidelines, etc.). An important role will be to establish 3Rs collaborations between the different National Contact Points (NCPs) belonging to parallel structures, e.g. PARERE Network (Preliminary Assessment of Regulatory Relevance) and EU-NETVAL, as well as with NGOs and trade associations. The WG on Education \& Training will aim to identify existing educational programs in Romania that include 3Rs knowledge, help to update existing materials and initiate development of new educational materials (e.g., books, handbooks, etc.), initiate permanent academic courses and programs (e.g., Master degrees), organize seminars and trainings on in vitro and in silico tools, host trainings on laboratory animal biology and welfare, etc. The WG on Research will focus on identification and participation in national, European and international grant proposals as well as in creating partnerships with industry in order to develop a program on knowledge transfer between academia and industry.

In conclusion, it was underlined that ROCAM's main goal should be the promotion of alternative methods and 3Rs approaches, their application in industry and their acceptance by regulators in Romania. ROCAM should play an important role in dissemination of $3 \mathrm{Rs}$ approaches, should facilitate training and educational programs in the area of $3 \mathrm{Rs}$ and should support research activities for the development, optimization, validation and application of alternative methods. Finally, ROCAM should act as a national main contact point in Romania in relation with other international 3Rs Centers. However, this process requires a close collaboration between different national key actors, thus ROCAM needs to initiate and form a national collaborative 3 Rs network of institutions active in this area in order to achieve its goals. Furthermore, a stronger active involvement of national representative at the level of European working groups and committees is needed in order to support even more these efforts, and to bring forward local and regional initiatives. This can only be possible if resources are sustainable. Therefore, ROCAM establishment envisages filling in some existing gaps in the region and to further support and promote 3 Rs developments.

\section{Correspondence to}

Prof. Carmen Socaciu, PhD

Vice Rector for Research and Innovation

University of Agricultural Sciences and Veterinary Medicine

Calea Manastur No. 3-5, 400372 Cluj-Napoca, Romania

e-mail: carmen.socaciu@usamvcluj.ro

http://rocam.usamvcluj.ro/ 